\title{
Homogeneous age dating of 55 Galactic globular clusters
}

\section{Clues to the Galaxy formation mechanisms}

\author{
M. Salaris ${ }^{1,2}$ and A. Weiss ${ }^{2}$ \\ 1 Astrophysics Research Institute, Liverpool John Moores University, Twelve Quays House, Egerton Wharf, \\ Birkenhead CH41 1LD, UK \\ 2 Max-Planck-Institut für Astrophysik, Karl-Schwarzschild-Str. 1, 85748 Garching, Germany
}

Received 21 February 2002 / Accepted 8 April 2002

\begin{abstract}
We present homogeneous age determinations for a large sample of 55 Galactic globular clusters, which constitute about $30 \%$ of the total Galactic population. A study of their age distribution reveals that all clusters from the most metal poor ones up to intermediate metallicities are coeval, whereas at higher $[\mathrm{Fe} / \mathrm{H}]$ an age spread exists, together with an age-metallicity relationship. At the same time, all clusters within a certain galactocentric distance appear coeval, whereas an age spread is present further away from the Galactic centre, without any correlation with distance. The precise value of $[\mathrm{Fe} / \mathrm{H}]$ and galactocentric distance for the onset of the age spread and the slope of the age-metallicity relationship are strongly affected by the as yet uncertain $[\mathrm{Fe} / \mathrm{H}] \mathrm{scale}$. We discuss how differences in the adopted $[\mathrm{Fe} / \mathrm{H}]$ scale and cluster sample size may explain discrepant results about the clusters age distribution reached by different authors. Taking advantage of the large number of objects included in our sample, we also tested the possibility that age is the global second parameter which determines the Horizontal Branch morphology, and found indications that age could explain the global behaviour of the second parameter effect.
\end{abstract}

Key words. Galaxy: formation - Galaxy: halo - globular clusters: general - stars: Hertzsprung-Russell diagram stars: Population II

\section{Introduction}

Less than a decade ago the age of the oldest globular clusters appeared to be much higher than that of the expanding universe. But at the end of the last millenium significant improvements both in models and in observational data, notably in the determination of cluster distances by virtue of Hipparcos-based distances, lead to a reduction of cluster ages. Presently, most determinations scatter around a typical age of the oldest objects of 12-14 Gyr (Salaris \& Weiss 1998; Carretta et al. 2000; VandenBerg 2000; Chaboyer 2001). With the growing confidence in the absolute age determinations and an increasing number of extensive homogeneous and high-quality photometric cluster data, the interest has shifted to questions concerning relative ages in order to learn about the formation of the galaxy and its halo and disk components.

In our own work (Salaris et al. 1997; Salaris \& Weiss 1997; Salaris \& Weiss 1998) we have used a method (to be fully described in Sect. 2), which is a mixture of absolute and relative age determinations in four metallicity ranges (see also Richer et al. 1996 for a similar technique). It does

Send offprint requests to: M. Salaris,

e-mail: ms@astro.livjm.ac.uk not need the knowledge of cluster distances, but instead predicts them. To determine the absolute ages of a sample of reference clusters for the various metallicity ranges, we use the brightness difference between horizontal branch (HB) and turn off (TO); the HB sets the cluster distances and the TO (whose brightness is the best predicted quantity to be used as age indicator) their age. For relative ages with respect to reference clusters we consider the differences in the extension of the subgiant branch, i.e., the colour range between the TO and the base of the red giant branch (RGB). In this way we are able to determine ages also in case of clusters for which the HB brightness determination is problematic (e.g., blue and/or sparsely populated HB). Since the differential properties of the TORGB colour difference across the entire cluster metallicity range is in principle subject to current uncertainties in convection treatment and colour transformations, the method works best (i.e., the uncertainties are minimized) if restricted to small $[\mathrm{Fe} / \mathrm{H}]$ ranges. When selecting the limits of the individual $[\mathrm{Fe} / \mathrm{H}]$ intervals, one must strike a balance between having a sizable number of clusters, and at the same time covering a $[\mathrm{Fe} / \mathrm{H}]$ interval where the predicted variation of the derivative of the TO-RGB colour difference with respect to age and $[\mathrm{Fe} / \mathrm{H}]$ is small. 
In Salaris \& Weiss (1998) we applied this method to a sample of 31 clusters taken from various sources, such that we could start to address the question of agemetallicity and age-galactocentric distance relations. This cluster sample appeared still too small to draw statistically meaningful conclusions; strictly speaking, relative ages are valid only within each metallicity bin, but we have shown in Salaris \& Weiss (1998) and Pulone et al. (1998) that they are consistent all over the $[\mathrm{Fe} / \mathrm{H}]$ range spanned by the clusters. In the present paper, we want to derive ages for a much larger sample of globular clusters by using our own isochrones and applying our preferred method. Relative ages for these clusters are a straightforward by-product.

There are other age determination techniques in use, some of them being easier to apply, but probably less accurate. All, of course, depend at some stage on theoretical isochrones. Semi-empirical methods (Buonanno et al. 1998; Rosenberg et al. 1999) are of particular interest for relative age-dating; they make extensive use of the colour difference between TO and RGB across all the $[\mathrm{Fe} / \mathrm{H}]$ range spanned by the galactic globular clusters, thus avoiding the necessity of RR Lyrae stars or a welldeveloped HB for distance determination. This allows to apply the method to a much larger number of clusters, and thus to obtain a solid picture of cluster formation throughout the Galactic history. The calibration of the TO-RGB colour difference with respect to age and metallicity is obtained empirically making use of clusters of different ages and $[\mathrm{Fe} / \mathrm{H}]$, for which reliable ages from the TO-HB brightness difference can be determined. Buonanno et al. (1998) noticed inconsistencies in relative cluster ages derived from brightness differences (between $\mathrm{HB}$ and TO) or colour differences as predicted by the isochrones they employed. In Pulone et al. (1998) we could demonstrate the self-consistency of our own isochrones for $(B-V)$-colours.

Following on this, Rosenberg et al. (1999) applied the semi-empirical method for colour differences in $(V-I)$ to their own large sample of cluster data (Rosenberg et al. 2000a,b). Their main result, based on the isochrones by Straniero et al. (1997) and a pre-release of VandenBerg et al. (2000) models, was that clusters of $[\mathrm{Fe} / \mathrm{H}] \leq-1.2$ are probably coeval (within $1 \mathrm{Gyr}$ ) and that at higher metallicities, up to $[\mathrm{Fe} / \mathrm{H}] \leq-1.0$ a few clusters younger by $\approx 4$ Gyr exist, too, and finally, that at $[\mathrm{Fe} / \mathrm{H}] \approx-0.7$ younger clusters prevail. However, if the brightness difference between HB (whose absolute magnitude was taken from the empirical relation by Carretta et al. 2000) and TO was used as the age indicator, this picture was not reproduced completely (some small differences in the age distribution for the most metal poor and most metal rich clusters), and, most importantly, the absolute age calibration for both relative age scales differed by up to 3 Gyr. A further set of isochrones tested (Cassisi et al. 1998) showed a large age spread for the colour-difference method. Evidently, semi-empirical methods for relative cluster ages spanning the whole metallicity range reveal different and internally inconsistent results for different isochrone sets, at least when one does not consider the HB brightness from ZAHB models consistent with the employed isochrones.

In this paper we will apply all these methods to a cluster sample which is a combination of the Rosenberg et al. (2000a,b) set (35 clusters) and 20 additional clusters with accurate photometry obtained recently. For this, we need colour transformations to $(V-I)$; in Weiss \& Salaris (1999) we investigated the properties of our own choice of transformations, showing that for selected clusters with photometry in $V, B$, and $I$ we can obtain identical ages and similar isochrone fitting quality for $V I$ and $B V$ photometric data. In the present paper we will determine cluster ages from VI-data using this and a second set of transformations (Alonso et al. 1999). We will demonstrate that the semi-empirical relative ages across the whole cluster metallicity range we obtain from the TO-RGB colour difference depend strongly on the chosen transformations; those by Alonso et al. (1999) provide results consistent with the ages inferred from the TO-HB brightness differences and the method employed in Salaris \& Weiss (1998), while the transformations used in Weiss \& Salaris (1999) are not suited for this purpose.

On the other hand, as will become evident, our preferred age determination method, as used by Salaris \& Weiss (1998), turns out to be largely insensitive to the specific colour transformation adopted, although it does make use of the colour difference between TO and RGB within restricted metallicity ranges. Similarly, the use of either $V I$ or $B V$-data does not influence the picture of the absolute and relative ages, but may affect only some individual clusters. Quantitatively, the effect is comparable to that of using different photometric sources; it is important to realize that in spite of the large observational progress and effort, the source of photometric data still influences individual and global results on cluster ages.

The paper is organized as follows: in the next section, our preferred age determination method and its application to a large sample of 55 globular clusters will be presented. This is, to our knowledge, the largest sample of clusters ever investigated, using the same age-dating method and theoretical isochrones. The sample size is a significant increase over the 43 clusters by Chaboyer et al. (1996); analysis of large clusters sample have been recently performed also by Richer et al. (1996, 36 objects), Buonanno et al. (1998, 33 objects) and Rosenberg et al. (1999, 35 objects). Our sample is largely based on homogeneous and very recent photometries; the isochrones and colour transformations are of the latest generation as well. In Sect. 3 we will then turn to the semi-empirical relative-age method using $(V-I)$-colours. We will present completely consistent results that agree well with those of Sect. 2. The discussion in Sect. 4 will analyze the age distribution of our cluster sample in light of the proposed scenarios of Galaxy formation. We will present also an analysis of the effect of the sample size and the adopted clusters metallicities on the derived age distribution. About the latter point, we have considered cluster $[\mathrm{Fe} / \mathrm{H}]$ 
values derived by both Carretta \& Gratton (1997, our reference metallicities) and Zinn \& West (1984). The two sets of values show differences up to $\sim 0.3$ dex (Carretta \& Gratton $1997[\mathrm{Fe} / \mathrm{H}]$ being higher) for intermediate metallicity clusters; a quadratic relationship (Carretta \& Gratton 1997) transforms Zinn \& West (1984) metallicities into the corresponding Carretta \& Gratton (1997) ones. The possibility that age is the global second parameter responsible (together with $[\mathrm{Fe} / \mathrm{H}]$ ) of the cluster $\mathrm{HB}$ morphology will be also tested. A short summary of the main results follows in Sect. 5 .

\section{The cluster sample and age-dating method}

Our GC sample consists of 55 objects. The $V$ magnitude difference between Zero Age $\mathrm{HB}$ (ZAHB) and TO, $\Delta V$, and the $(V-I)$ colour difference (VI Johnson-Cousins bands) between $\mathrm{TO}$ and the base of the RGB, $\Delta(V-I)$ as defined in Rosenberg et al. (1999, R99), for 35 of them are taken from the homogeneous database by R99. For the remaining 20 objects $\Delta V, \Delta(V-I)$ or $\Delta(B-V)$, the corresponding colour difference between TO and base of the RGB in $(B-V)$ as defined by VandenBerg et al. (1990), are taken from Salaris \& Weiss (1998, SW98) and other papers published in the last 5 years (see Table 1).

The age-dating method is analogous to the procedure outlined in Salaris \& Weiss (1997, SW97) and SW98. The GC sample is divided into four metallicity intervals, which are the same ones as in SW98, apart from the last interval; this we extended to include in the same group the thick disk clusters present in the sample. The metallicity intervals therefore are $-2.15 \leq[\mathrm{Fe} / \mathrm{H}] \leq-1.75$, $-1.74 \leq[\mathrm{Fe} / \mathrm{H}] \leq-1.3,-1.29 \leq[\mathrm{Fe} / \mathrm{H}] \leq-0.9$, and $-0.89 \leq[\mathrm{Fe} / \mathrm{H}] \leq-0.6$. We used the $[\mathrm{Fe} / \mathrm{H}]$ values given by Rutledge et al. (1997) on the Carretta \& Gratton (1997, CG97) scale (the internal accuracy of these $[\mathrm{Fe} / \mathrm{H}]$ values is of the order of $0.10 \mathrm{dex}$ ); this introduces small differences with respect to the $[\mathrm{Fe} / \mathrm{H}]$ values used by $\mathrm{SW} 98$ which were either directly from CG97 or transformed from the Zinn \& West (1984, ZW84) scale using the relationship given by CG97, but allows us to directly compare our results with $\mathrm{R} 99$, who selected the same $[\mathrm{Fe} / \mathrm{H}]$ values for their sample. In case of clusters without estimates from Rutledge et al. (1997), but with estimates on the ZW84 scale, we again transformed their $[\mathrm{Fe} / \mathrm{H}]$ onto the CG97 scale using the conversion formula given by CG97.

Within each interval a reference cluster showing a $\mathrm{HB}$ well populated at the instability strip region and/or at its red side was selected; this allows a reliable determination of the ZAHB level to be obtained, and the absolute age to be determined accurately from $\Delta V$, by comparing it with $\Delta V$ taken from the isochrone for the metallicity under consideration. The reference clusters are, respectively, M 15 (NGC 7078), M 3 (NGC 5272), NGC 6171 and 47 Tuc (NGC 104). In our new analysis M 15 and M 3 have replaced M 68 (NGC 4590) and NGC 6584 as reference clusters in their respective groups (SW98). Due to the small $[\mathrm{Fe} / \mathrm{H}]$ differences with respect to SW98, the cluster NGC 6171 has now moved to the third metallicity group where it has replaced M 5 (NGC 5904) as the reference cluster. For all the four reference clusters we used $\Delta V$ values taken from R99.

Once the absolute ages of the reference clusters were obtained, the age of all other clusters within each group was determined differentially with respect to the reference one, by using the differences in $\Delta(V-I)$ to derive differences in age, again by using the isochrones. In case of clusters with $B V$ photometry, their relative age with respect to the template clusters was obtained from $\Delta(B-V)$. Because of the approach of tying relative ages within limited metallicity ranges to the absolute age of selected reference clusters, we will, for the remainder of this paper, call our method simply the $A M$-method.

We have employed in our analysis the $B V$ and $V I$ isochrones described in SW98 and Weiss \& Salaris (1999), assuming a cosmological helium mass fraction $Y=0.230$, an average $\alpha$-element overabundance $[\alpha / \mathrm{Fe}]=0.4$, and a helium enrichement factor $\Delta Y / \Delta Z=3$. For the $(V-I)$ colours we use a combination of the transformations by Bessell et al. (1998) for the main sequence and von Braun et al. (1998) for the RGB, as described in Weiss \& Salaris (1999). For further details, see the original papers (SW98, Weiss \& Salaris 1999). We briefly recall here that the ZAHB luminosities from our models, which set the zero point of our absolute ages, are in agreement with the ZAHB brightness levels obtained by Carretta et al. (2000) from the subdwarf fitting method applied to a sample of Galactic globular clusters. They are therefore about 0.13 mag brighter than the relationship used by R99, which results from an average of the subdwarf fitting distances together with other distance determinations, discussed in Carretta et al. (2000).

Our procedure allows a reliable determination of the age distribution with respect to the absolute age of the template clusters, largely unaffected by uncertainties in the convection treatment, by the precise value of the cluster metallicity, and by the inclusion of element diffusion (see the discussion in SW97). It is also fairly independent of current uncertainties in colour transformations. This we verified by repeating the procedure for the clusters with VI photometry using a different set of RGB colour transformations together with our isochrones (Alonso et al. 1999); we find only modest age changes within \pm 0.6 Gyr. A more detailed discussion on the issue of colour transformations will be presented in the next section.

Two further tests have been performed in order to assess the reliability of our age determination: in the first one we have simply moved clusters close to the boundary of two contiguous groups (e.g., NGC 6254, NGC 3201, NGC 6397) to the adjacent one and redetermined their age with respect to the absolute age of the new template cluster. The resulting absolute ages agree within 0.3 Gyr with the ones obtained from the original group location. In the second test, we have derived the age of all 35 clusters from R99 making use of their estimated $\Delta V$-values for the whole sample. Even in case of blue 


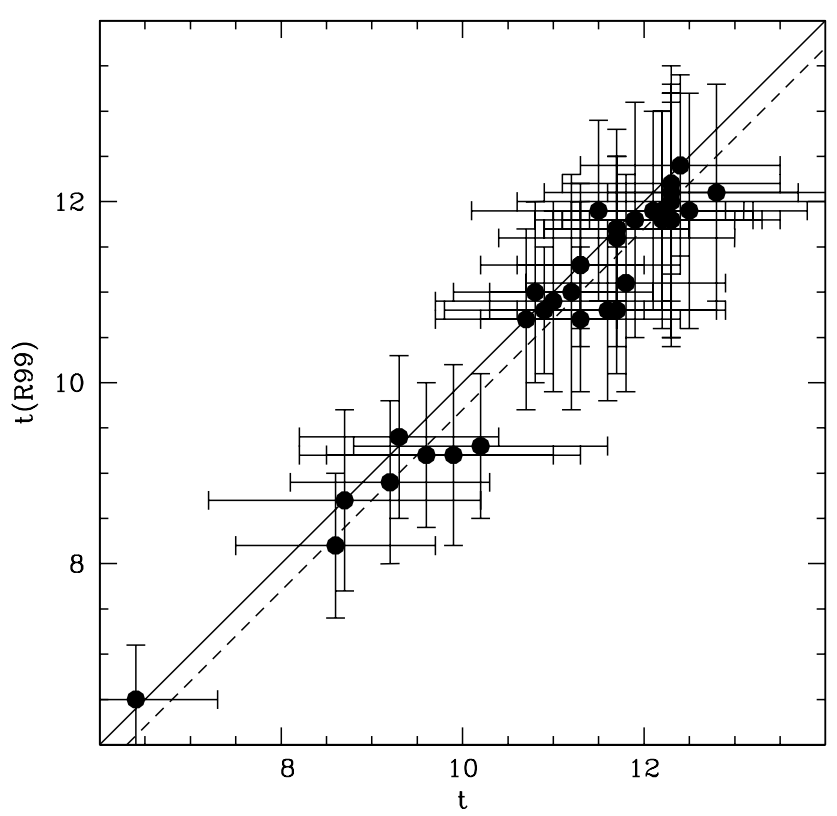

Fig. 1. Comparison of the absolute ages (in Gyr) obtained using the $\Delta V$ values provided by R99 for their whole sample (vertical axis), with the ages obtained with our AM-method (horizontal axis). Errors are as shown in Table 1, or as given by R99. The solid line represents the locus of equal ages; dashed line corresponds to the average difference between the two sets of ages (0.3 Gyr).

HB morphologies, R99 have derived the level of the ZAHB extrapolating the observed ZAHB location towards the red, by fitting template cluster HBs uniformly populated to the observed branches. While we consider this approach being less accurate then in cases with observed HBs populated at the instability strip or at its red side, it is of course a valid estimate of the ZAHB brightness. The resulting ages for the 35 clusters, displayed in Fig. 1, indeed agree extremely well with the values obtained with the AM-technique, with an average difference of only $0.3 \mathrm{Gyr}$ ( $\Delta V$ ages being younger). This test confirms the mutual consistency of the $\Delta V$ relative ages and those obtained by the AM-method over the whole $[\mathrm{Fe} / \mathrm{H}]$ range spanned by the cluster sample.

A similar test, but in $(B-V)$, was performed for two individual clusters, NGC 6712 and NGC 6934. BV photometry is available for both of them, and both have extremely well-defined ZAHB levels. We could therefore derive their absolute age also directly from $\Delta V$. As in the case of the R99 cluster sample with $V I$ photometry, the results are consistent, within $0.4 \mathrm{Gyr}$, with the relative age obtained from their $\Delta(B-V)$ with respect to the corresponding template cluster.

The final results for the ages of the entire sample are displayed in Table 1 . In case of non-template clusters, the final error in the absolute age has been derived by adding in quadrature the error in the age of the corresponding template cluster (which is determined by the errors in $\Delta V$ and the internal errors in the adopted metallicity scale) to the error in the relative age estimate (which is determined

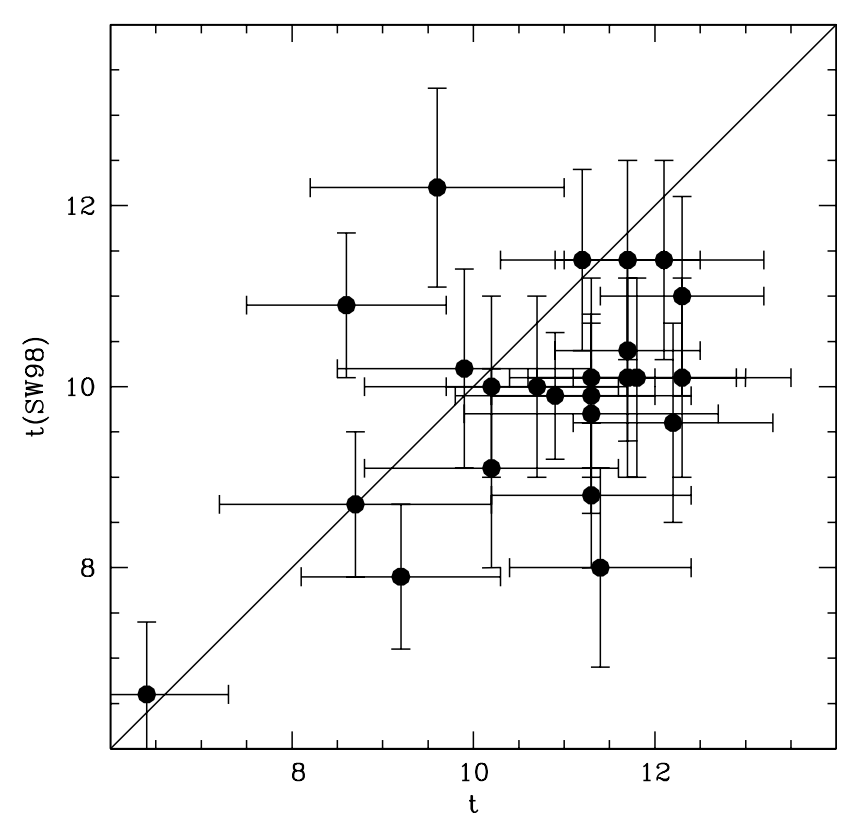

Fig. 2. Comparison of the absolute ages (in Gyr) obtained by SW98 (vertical axis) with the values found in the present work (horizontal axis), in case of 24 common clusters for which we have used new photometric data. The solid line represents the locus of equal ages.

by the errors in the differences in $\Delta(V-I)$ or $\Delta(B-V)$, and the internal errors in the adopted metallicity scale).

There are 24 clusters in common with the SW98 sample, for which we have now employed more recent and more homogeneous photometry (mainly in the VI bands); 21 of these clusters belong to the R99 sample. In Fig. 2 we compare the absolute ages derived by SW98 (where essentially the same $[\mathrm{Fe} / \mathrm{H}]$ scale was employed) and the ones we now obtained for these 24 clusters in common. There is a clear average shift towards higher values in our new sample, which is due to the new photometric data; the average difference is of the order of 1 Gyr. However, in case of two metal-rich clusters, namely NGC 6366 and NGC 1261 (new VI photometries from R99), we get ages considerably lower by $\sim 2.5$ Gyr.

Figure 3 displays the absolute ages as a function of $[\mathrm{Fe} / \mathrm{H}]$ and galactocentric distance $\left(R_{\mathrm{gc}}\right)$ for our whole sample, as reported in Table 1. Filled circles correspond to clusters in the R99 sample, while open squares denote the remaining ones. There are no significant differences between these two sub-samples (see also Sect. 4). In case of the clusters' $R_{\mathrm{gc}}$ we have adopted, as a reference, the values provided by Harris (1996). Strictly speaking, $R_{\mathrm{gc}}$ depends on the cluster distance modulus, but for differences of the order of 0.1-0.15 mag around the Harris (1996) values, $R_{\mathrm{gc}}$ is not changed appreciably, and in case of, e.g., the 35 clusters with ZAHB levels from R99, we determined distance moduli within this range from the Harris (1996) values.

It is interesting to determine the age difference for two classic second-parameter pairs, namely NGC 288NGC 362 and M 3-M 13 (NGC 6205), both contained 
Table 1. Data for the 55 clusters analyzed. The columns display, respectively, cluster name, age using the CG97 scale and associated error, $[\mathrm{Fe} / \mathrm{H}]$ on the CG97 scale, age using the ZW84 scale and associated error, $[\mathrm{Fe} / \mathrm{H}]$ on the ZW84 scale, galactocentric distance, HB type, photometric bands used for the age determination, source of the data (see text for details). Reference clusters are emphasized by bold type characters.

\begin{tabular}{|c|c|c|c|c|c|c|c|c|}
\hline Cluster & 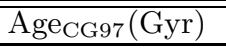 & {$[\mathrm{Fe} / \mathrm{H}]_{\mathrm{CG} 97}$} & "Agezw84(Gyr) & {$[\mathrm{Fe} / \mathrm{H}]_{\text {ZW84 }}$} & $R_{\mathrm{gc}}(\mathrm{Kpc})$ & $\mathrm{HB}_{\text {type }}$ & phot & source $^{a}$ \\
\hline NGC 104 & $10.7 \pm 1.0$ & -0.78 & $10.7 \pm 1.0$ & -0.71 & 7.4 & -0.99 & $V I$ & R99 \\
\hline NGC 288 & $11.3 \pm 1.1$ & -1.14 & $11.9 \pm 1.1$ & -1.40 & 11.6 & 0.98 & $V I$ & R99 \\
\hline NGC 362 & $8.7 \pm 1.5$ & -1.09 & $9.5 \pm 1.5$ & -1.33 & 9.3 & -0.87 & $V I$ & R99 \\
\hline NGC 1261 & $8.6 \pm 1.1$ & -1.08 & $9.1 \pm 1.1$ & -1.32 & 18.2 & -0.71 & $V I$ & R99 \\
\hline NGC 1851 & $9.2 \pm 1.1$ & -1.03 & $9.1 \pm 1.1$ & -1.23 & 16.7 & -0.36 & $V I$ & R99 \\
\hline NGC 1904 & $11.7 \pm 1.3$ & -1.37 & $12.6 \pm 1.3$ & -1.67 & 18.8 & 0.89 & $V I$ & R99 \\
\hline NGC 2298 & $12.6 \pm 1.4$ & -1.71 & $12.9 \pm 1.4$ & -1.85 & 15.7 & 0.93 & $V I$ & Т01 \\
\hline NGC 2419 & $12.3 \pm 1.0$ & -2.14 & $12.8 \pm 1.0$ & -2.10 & 91.5 & 0.86 & $V I$ & H97 \\
\hline NGC 2808 & $9.3 \pm 1.1$ & -1.11 & $10.2 \pm 1.1$ & -1.36 & 11.0 & -0.49 & $V I$ & R99 \\
\hline NGC 3201 & $11.3 \pm 1.1$ & -1.24 & $12.1 \pm 1.1$ & -1.53 & 9.0 & 0.08 & $V I$ & R99 \\
\hline NGC 4590 & $11.2 \pm 0.9$ & -2.00 & $11.2 \pm 0.9$ & -2.11 & 10.1 & 0.17 & $V I$ & R99 \\
\hline NGC 5053 & $10.8 \pm 0.9$ & -1.98 & $10.8 \pm 0.9$ & -2.10 & 16.9 & 0.52 & $V I$ & R99 \\
\hline NGC 5272 & $11.3 \pm 0.7$ & -1.33 & $12.1 \pm 0.7$ & -1.66 & 12.2 & 0.08 & $V I$ & R99 \\
\hline NGC 5466 & $12.2 \pm 0.9$ & -2.13 & $12.5 \pm 0.9$ & -2.22 & 17.2 & 0.58 & $V I$ & R99 \\
\hline NGC 5897 & $12.3 \pm 1.2$ & -1.73 & $12.4 \pm 1.2$ & -1.93 & 7.7 & 0.86 & $V I$ & R99 \\
\hline NGC 5904 & $10.9 \pm 1.1$ & -1.12 & $11.6 \pm 1.1$ & -1.38 & 6.2 & 0.31 & $V I$ & R99 \\
\hline NGC 6093 & $12.4 \pm 1.1$ & -1.47 & $12.9 \pm 1.1$ & -1.75 & 3.8 & 0.93 & $V I$ & R99 \\
\hline NGC 6101 & $10.7 \pm 1.4$ & -1.76 & $11.0 \pm 1.4$ & -1.81 & 11.1 & 0.84 & $B V$ & SDC91 \\
\hline NGC 6121 & $11.7 \pm 1.1$ & -1.05 & $11.9 \pm 1.1$ & -1.27 & 5.9 & -0.06 & $V I$ & R99 \\
\hline NGC 6171 & $11.7 \pm 0.8$ & -0.95 & $11.7 \pm 0.8$ & -1.09 & 3.3 & -0.73 & $V I$ & R99 \\
\hline NGC 6205 & $11.9 \pm 1.1$ & -1.33 & $13.0 \pm 1.3$ & -1.63 & 8.7 & 0.97 & $V I$ & R99 \\
\hline NGC 6218 & $12.5 \pm 1.3$ & -1.14 & $12.7 \pm 1.3$ & -1.40 & 4.5 & 0.97 & $V I$ & R99 \\
\hline NGC 6254 & $11.8 \pm 1.1$ & -1.25 & $12.2 \pm 1.1$ & -1.55 & 4.6 & 0.98 & $V I$ & R99 \\
\hline NGC 6341 & $12.3 \pm 0.9$ & -2.10 & $12.8 \pm 0.9$ & -2.24 & 9.6 & 0.91 & $V I$ & R99 \\
\hline NGC 6352 & $9.9 \pm 1.4$ & -0.70 & $9.7 \pm 1.4$ & -0.50 & 3.3 & -1.00 & $V I$ & R99 \\
\hline NGC 6362 & $11.0 \pm 1.3$ & -0.99 & $11.1 \pm 1.3$ & -1.08 & 5.3 & -0.58 & $V I$ & R99 \\
\hline NGC 6366 & $9.6 \pm 1.4$ & -0.73 & $9.4 \pm 1.4$ & -0.58 & 5.0 & -0.97 & $V I$ & R99 \\
\hline NGC 6397 & $12.1 \pm 1.1$ & -1.76 & $12.5 \pm 1.1$ & -1.94 & 6.0 & 0.98 & $V I$ & R99 \\
\hline NGC 6426 & $12.9 \pm 1.0$ & -2.11 & $13.0 \pm 1.0$ & -2.20 & 14.2 & 0.53 & $V I$ & H99 \\
\hline NGC 6535 & $12.8 \pm 1.2$ & -1.51 & $13.1 \pm 1.2$ & -1.78 & 3.9 & 1.00 & $V I$ & R99 \\
\hline NGC 6584 & $11.3 \pm 1.4$ & -1.30 & $12.1 \pm 1.6$ & -1.54 & 7.0 & -0.15 & $B V$ & SF95 \\
\hline NGC 6624 & $10.6 \pm 1.4$ & -0.70 & $10.6 \pm 1.4$ & -0.50 & 1.2 & -1.00 & $V I$ & H0O \\
\hline NGC 6637 & $10.6 \pm 1.4$ & -0.78 & $10.6 \pm 1.4$ & -0.72 & 1.6 & -1.00 & $V I$ & $\mathrm{H} 00$ \\
\hline NGC 6652 & $11.4 \pm 1.0$ & -0.81 & $11.4 \pm 1.0$ & -0.89 & 2.4 & -1.00 & $V I$ & $\mathrm{C} 00$ \\
\hline NGC 6656 & $12.3 \pm 1.2$ & -1.41 & $12.5 \pm 1.2$ & -1.75 & 4.9 & 0.91 & $V I$ & R99 \\
\hline NGC 6681 & $11.5 \pm 1.4$ & -1.35 & $11.9 \pm 1.4$ & -1.51 & 2.1 & 0.96 & $V I$ & R99 \\
\hline NGC 6712 & $10.4 \pm 1.4$ & -0.94 & $10.5 \pm 1.4$ & -1.07 & 3.5 & -0.64 & $B V$ & P01 \\
\hline NGC 6723 & $11.6 \pm 1.3$ & -0.96 & $11.6 \pm 1.3$ & -1.12 & 2.6 & -0.08 & $V I$ & R99 \\
\hline NGC 6752 & $12.2 \pm 1.1$ & -1.24 & $12.7 \pm 1.1$ & -1.54 & 5.2 & 1.00 & $V I$ & R99 \\
\hline NGC 6779 & $12.3 \pm 1.4$ & -1.61 & $12.8 \pm 1.4$ & -1.94 & 9.7 & 0.98 & $V I$ & R99 \\
\hline NGC 6809 & $12.3 \pm 1.7$ & -1.54 & $12.4 \pm 1.7$ & -1.80 & 3.8 & 0.87 & $V I$ & R99 \\
\hline NGC 6838 & $10.2 \pm 1.4$ & -0.73 & $10.1 \pm 1.4$ & -0.58 & 6.7 & -1.00 & $V I$ & R99 \\
\hline NGC 6934 & $9.6 \pm 1.5$ & -1.30 & $10.0 \pm 1.6$ & -1.54 & 14.3 & 0.25 & $B V$ & P99 \\
\hline NGC 7078 & $11.7 \pm 0.8$ & -2.02 & $11.8 \pm 0.8$ & -2.13 & 10.4 & 0.67 & $V I$ & R99 \\
\hline NGC 7099 & $11.9 \pm 1.4$ & -1.92 & $12.3 \pm 1.4$ & -2.05 & 7.1 & 0.89 & $B V$ & RFV88 \\
\hline NGC 7492 & $12.0 \pm 1.4$ & -1.41 & $12.1 \pm 1.4$ & -1.51 & 24.9 & 0.81 & $B V$ & CRF91 \\
\hline Arp 2 & $11.3 \pm 1.4$ & -1.45 & $11.5 \pm 1.4$ & -1.84 & 21.4 & 0.86 & $B V$ & B98 \\
\hline Eridanus & $8.9 \pm 1.6$ & -1.20 & $8.4 \pm 1.6$ & -1.48 & 95.2 & -1.00 & $V I$ & S99 \\
\hline IC 4499 & $12.1 \pm 1.4$ & -1.26 & $11.2 \pm 1.2$ & -1.50 & 15.7 & 0.11 & $B V$ & B98 \\
\hline Pal 3 & $9.7 \pm 1.3$ & -1.39 & $9.2 \pm 1.3$ & -1.57 & 95.9 & -0.50 & $V I$ & S99 \\
\hline Pal 4 & $9.5 \pm 1.6$ & -1.07 & $9.2 \pm 1.6$ & -1.58 & 111.8 & -1.00 & $V I$ & S99 \\
\hline Pal 5 & $9.8 \pm 1.4$ & -1.24 & $10.0 \pm 1.4$ & -1.47 & 18.6 & -0.40 & $B V$ & SM86 \\
\hline Pal 12 & $6.4 \pm 0.9$ & -0.83 & $6.4 \pm 0.9$ & -0.82 & 15.9 & -1.00 & $V I$ & R99 \\
\hline Rup 106 & $10.2 \pm 1.4$ & -1.49 & $10.4 \pm 1.4$ & -1.90 & 18.5 & -0.82 & $B V$ & B98 \\
\hline Terzan 7 & $7.4 \pm 1.4$ & -0.56 & $7.5 \pm 1.4$ & -1.00 & 16.0 & -1.00 & $B V$ & B98 \\
\hline
\end{tabular}

${ }^{a}$ Testa et al. (2001 T01), Harris et al. (1997, H97), Sarajedini \& Da Costa (1991, SDC91), Hatzidimitriou et al. (1999, H99), Sarajedini \& Forrester (1995, SF95), Heasley et al. (2000, H00), Chaboyer et al. (2000, C00), Paltrinieri et al. (2001, P01), Piotto et al. (1999, P99), Richer et al.(1988, RFV88), Cote et al. (1991, CRF91), Buonanno et al. (1998, B98), Stetson et al. (1999, S99), Smith et al. (1986, SM86). 

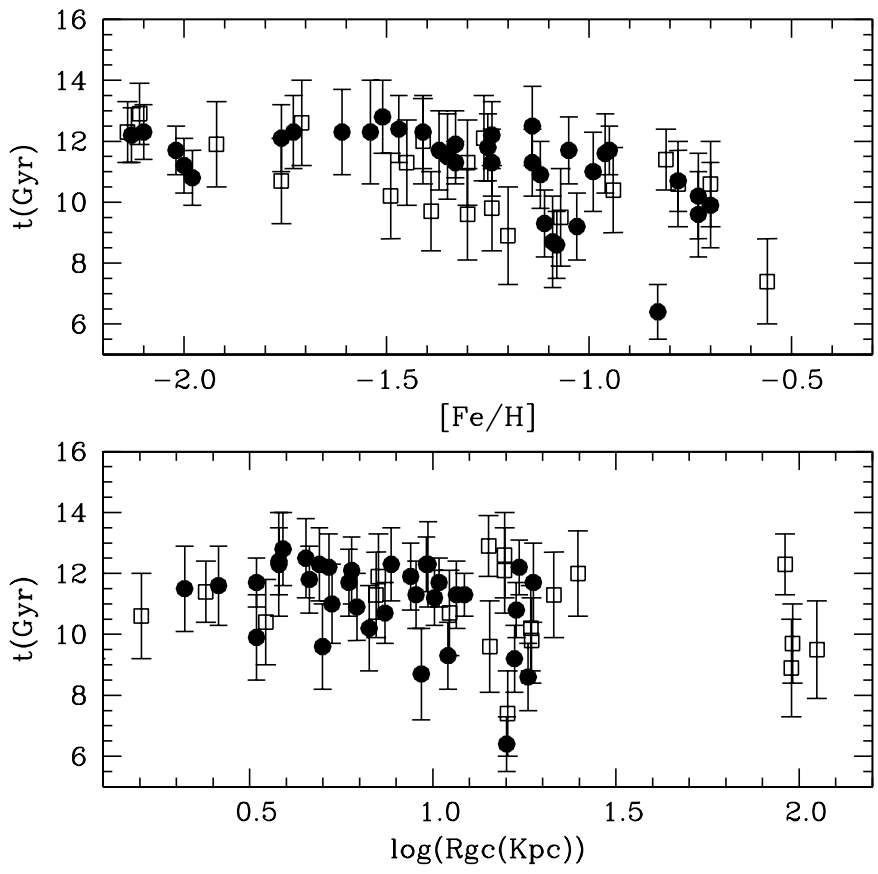

Fig. 3. Distribution of the absolute ages (in Gyr) as function of $[\mathrm{Fe} / \mathrm{H}]$ (upper panel) and $R_{\mathrm{gc}}$ (lower panel). Filled circles correspond to clusters in the R99 sample, while open squares denote the remaining ones.
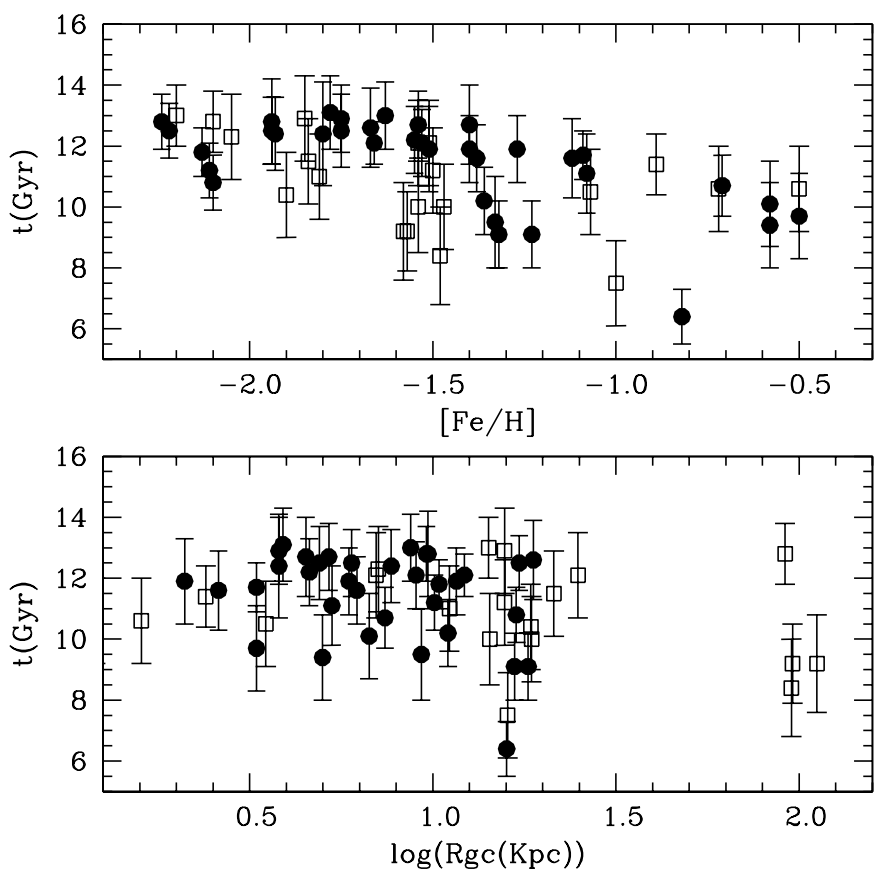

Fig. 4. As in Fig. 3, but assuming the ZW84 [Fe/H] scale for the clusters instead of the CG97 one in the previous figure.

in our sample. When deriving errors in age differences within the same metallicity group, we have just to take into account the error coming from the use of $\Delta(V-I)$ as the relative age indicator, without adding the contribution of the error in the absolute age of the template cluster (as is done in Table 1), because for typical absolute age errors of \pm 1 Gyr, the differential properties of $\Delta(V-I)$ as a function of metallicity and age are negligibly affected.

For the pair NGC 288-NGC 362 we obtain an age difference of $2.6 \pm 1.5$ Gyr (NGC 288 being older), in good agreement with the results by Bellazzini et al. (2001), but in contrast to SW97, where the two clusters appeared to be coeval. The reason for this change lies in the new photometric data. According to the simulations by Catelan et al. (2001), this age difference, coupled with the absolute ages we obtain and the use of the CG97 $[\mathrm{Fe} / \mathrm{H}]$ scale, can explain the overall different HB morphology; however, canonical HB models appear unable to reproduce the detailed morphology of the red end of NGC 288 HB (Catelan et al. 2001).

In case of M 3-M 13 we obtain a difference of $0.6 \pm$ 1.0 Gyr (M13 older), much less significant than the $1.7 \pm$ $0.7 \mathrm{Gyr}$ as obtained by Rey et al. (2001) from their $B V$ photometry. M 13 was not contained in our previous work. The same negligible age difference we obtain from the $\Delta V$ values derived by R99; it seems therefore that the discrepancy between our and Rey et al. (2001) result is due to real differences in the photometric data, and not to the use of different passbands for the TO-RGB colour differences and inconsistency in the colours of the theoretical isochrones. Problems with the calibration of the photometry may possibly lead to this kind of discrepancy. Rey et al. (2001) noticed, for example, that their derived fiducial line for M 13 agrees well with those obtained by Richer \& Fahlman (1986) and Yim et al. (2000), but differs in the main sequence and subgiant branch region from the fiducial by Paltrinieri et al. (1998).

It is also interesting to notice that Arp 2 and Rup 106 do not appear much younger than the bulk of the clusters at their metallicity; the reason why Buonanno et al. (1998) found higher age differences is mainly the fact that their adopted clusters' absolute ages are higher. As discussed in detail in, e.g., Pulone et al. (1998), lower absolute ages imply smaller age differences for a given observed distribution of $\Delta V, \Delta(B-V)$ or $\Delta(V-I)$ values. As discussed in SW98 there are preliminary indications that Rup 106 and also Pal 12 may not show $\alpha$-element enhancement. In this case, their ages displayed in Table 1 should be increased by about 1 Gyr.

To highlight the effect of the present uncertainties in the $[\mathrm{Fe} / \mathrm{H}]$ scale, we have also derived ages, as a test, by using the $[\mathrm{Fe} / \mathrm{H}]$ values given by Rutledge et al. (1997) on the ZW84 scale (internal accuracy again of the order of $0.10 \mathrm{dex}$ ), complemented, if needed, by data in the original ZW84 paper or coming from other spectral indices calibrated on the ZW84 scale. In this case the $[\mathrm{Fe} / \mathrm{H}]$ range spanned by our sample is larger than the CG97 scale. We have therefore divided the sample into 5 groups, having as template clusters M $15(-2.3 \leq[\mathrm{Fe} / \mathrm{H}] \leq-2.0)$, NGC $6656(-1.99 \leq[\mathrm{Fe} / \mathrm{H}] \leq-1.7)$, M $3(-1.69 \leq$ $[\mathrm{Fe} / \mathrm{H}] \leq-1.4)$, NGC $6171(-1.39 \leq[\mathrm{Fe} / \mathrm{H}] \leq-0.9)$ and 47 Tuc $(-0.89 \leq[\mathrm{Fe} / \mathrm{H}] \leq-0.5)$. Figure 4 shows the age distribution resulting from the AM-method as a function of $[\mathrm{Fe} / \mathrm{H}]$ and $R_{\mathrm{gc}}$, when using the ZW84 $[\mathrm{Fe} / \mathrm{H}]$ scale. 
Using this metallicity scale, the age differences between the two second parameter pairs discussed remain basically unchanged. In fact, in case of NGC 288-NGC 362 we obtain now a difference of $2.4 \pm 1.5 \mathrm{Gyr}$, and for M 3-M 13 we get $0.9 \pm 1.0$ Gyr. A detailed analysis of the age distributions displayed in Figs. 3 and 4 will be presented in the last section.

\section{Colour-based relative ages}

We consider it important that isochrones - together with colour transformations - yield ages largely independent of the age indicator. If consistency is achieved, one could then in principle apply different age indicators to different clusters, according to the quality of the available data, and still have consistent ages for all the sample.

In the last section we showed that the AM-method yields results which are consistent with absolute and relative ages from the $\Delta V$ - or vertical method. Another age indicator - the horizontal one - is the colour difference between TO and RGB, which we used already in the AM-method to obtain relative ages within each metallicity bin. In the following, we will call this quantity simply $\delta C$, independent of the actual colour it refers to. From an observational point of view this age-indicator is the easiest one to determine, since it uses a CMD portion which is common to all clusters, is well-populated, and thus for sufficiently deep photometry - observable with high accuracy. Reddening is not important, as long as it is low and not differential. However, the difficulties start when using it for absolute ages or over a wide range of metallicities; in this case, observed individual $\delta C$ values have to be compared to theoretically predicted ones, and colour transformations - with their known uncertainties - must be able to predict with extreme accuracy the absolute values of colours over a large $[\mathrm{Fe} / \mathrm{H}]$ and effective temperature range.

Because of the easy observational accessibility, Buonanno et al. (1998) developed a semi-empirical relative-age determination method using $\delta C$ in the $B V$ plane. It sets out by selecting a group of coeval clusters of all metallicities, which defines the empirical function $\delta C([\mathrm{Fe} / \mathrm{H}])$ at the age $t_{0}$ of this group. For this step, $\Delta V$ and theoretical isochrones are needed. It turns out that membership to the coeval group is rather independent of the isochrones used, but not the actual value for $t_{0}$. The result for our own isochrones is shown in Fig. 5. The empirical $\delta C([\mathrm{Fe} / \mathrm{H}])$-relation at $t_{0}$ can be compared to the theoretical one. Agreement is not necessarily given (Buonanno et al. 1998) and depends on isochrone set and colour transformation.

In the next step clusters of ages differing from $t_{0}$ are used to derive the relation $\delta C(t)$ at various $[\mathrm{Fe} / \mathrm{H}]$ points. Age differences are again determined from the vertical method and isochrones. Combined with the first step, one obtains $\delta C$ as a function of age and metallicity, such that the observed values for $\delta C$ along with $[\mathrm{Fe} / \mathrm{H}]$ yield the age straightforwardly. This makes the method very attractive

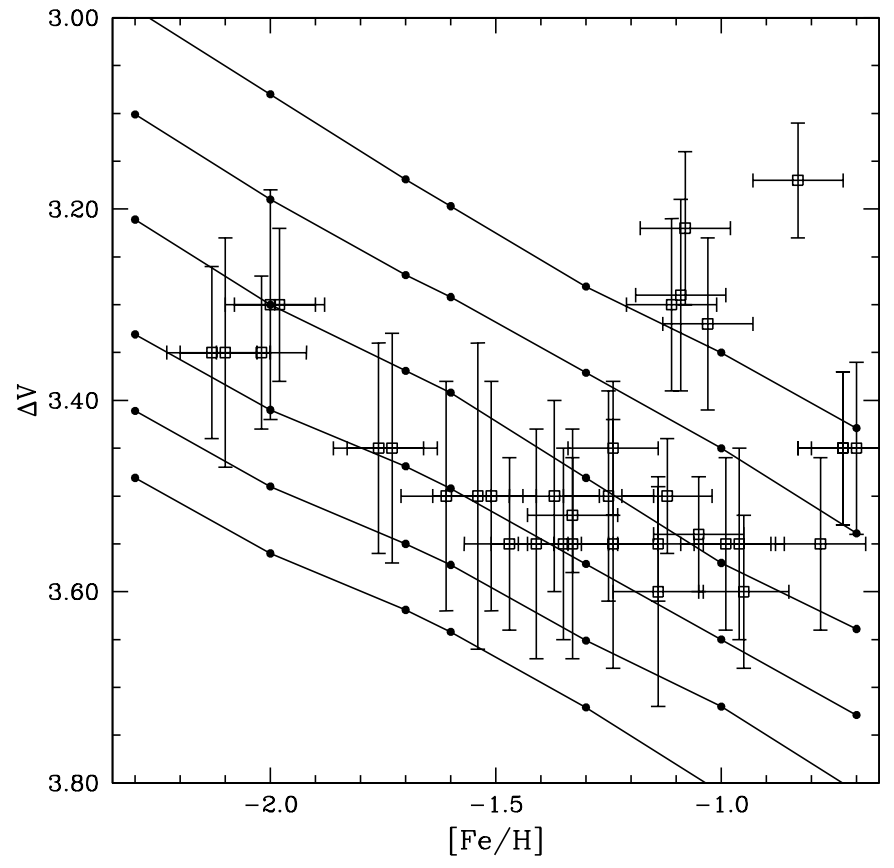

Fig. 5. Observed $\Delta V$ values for the 35 clusters from R99 along with the theoretical values from our isochrones, plotted for 14 9 Gyrs (bottom to top in steps of -1 Gyr).

for large cluster samples. Because of the problems mentioned above the method works best for relative ages, but one has to keep in mind that age differences also depend on the absolute age (SW97). In Pulone et al. (1998) we showed that our own isochrones and colour transformations to $(B-V)$ result in a $\delta C([\mathrm{Fe} / \mathrm{H}])$ relation consistent with observations and that relative ages from this method agree well with those we obtain from the AM-method, which might be understood as a "piecewise" application of that of Buonanno et al. (1998).

This same idea has then been used by R99 for their clusters observed in $V$ and $I$ (Rosenberg et al. 2000a,b). They employed isochrones from Straniero et al. (1997) and a pre-release of VandenBerg et al. (2000) models for the theoretical determination of $\delta C(t)$ at various $[\mathrm{Fe} / \mathrm{H}]$, and the TO brightness at various ages and $[\mathrm{Fe} / \mathrm{H}]$; as for the HB brightness, they considered the empirical ZAHB brightness relationship by Carretta et al. (2000), lacking access to ZAHB models consistent with the isochrones adopted.

As in Buonanno et al. (1998) the comparison of ages from $\Delta V$ and $\Delta(V-I)$ revealed inconsistencies: the absolute age $t_{0}$ of the coeval reference sample depends on age indicator and isochrones: it is (ages in Gyr) 14 for VandenBerg et al. (2000) and $\sim 15$ for Straniero et al. (1997), according to $\Delta V$, but, respectively, 16 and 13 for $\Delta(V-I)$, i.e. there are changes of $2 \mathrm{Gyr}$ with respect to the $\Delta V$ ages, but they are in opposite directions for the two isochrone sets.

We already demonstrated (Fig. 1) that the relative AM-ages, which are basically obtained from the horizontal method, agree well with the $\Delta V$ values for the R99 


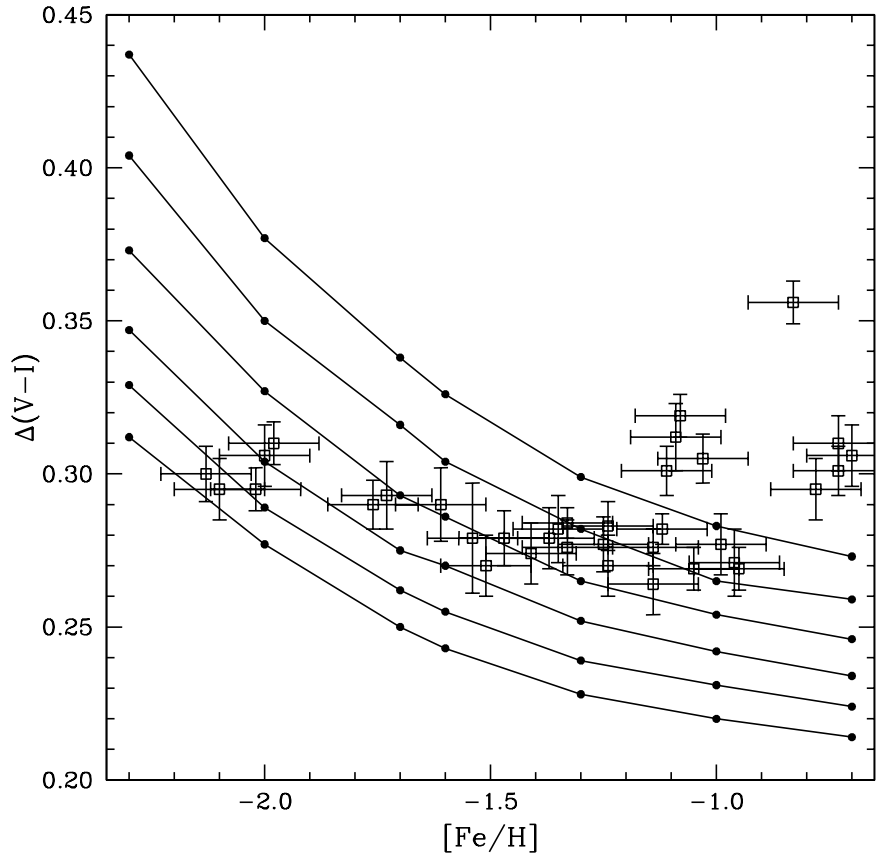

Fig. 6. The observed horizontal relative age indicator $\Delta(V-I)$ for the 35 clusters from R99 along with the theoretical values from our isochrones, plotted for 14-9 Gyrs (bottom to top in steps of $-1 \mathrm{Gyr})$. Isochrones were transformed to $(V-I)$ using the colour transformation selected in Weiss \& Salaris (1999).

sample. In particular, the majority of clusters is coeval at $\approx 12$ Gyr with a small metal-rich group at ages lower by 1-3 Gyr (see Fig. 5). According to the (global) horizontal age indicator $\Delta(V-I)$, however, there is a clear age increase of almost 3 Gyr within the coeval group (Fig. 6). This effect is most pronounced at the lowest metallicities. The $\Delta(V-I)$ therefore, when used as an age indicator across all the metallicity range, along with our isochrones and colour transformations, produces inconsistent results with respect to $\Delta V$. Note, however, that the horizontal average age of the "coeval" group is around 11 Gyr and within the errors - consistent with that of the vertical one (Fig. 5), and that the whole discrepancy in the $\Delta(V-I)$ relative ages is due to a variation in $\Delta(V-I)$ of only $\approx 0.05 \mathrm{mag}$. This is a strong indication that theoretical colours have to be very accurate for the whole metallicity range of globular clusters in order that this method can return reliable results.

To emphasize this point we show in Fig. 7 the predicted RGB colours for 12 Gyr-isochrones of different metallicities as obtained by using the transformations of Weiss \& Salaris (1999) or Alonso et al. (1999). Evidently, in the former case, which is based on von Braun et al. (1998), the dependence of colour on metallicity is non-monotonic and RGBs are piling up at a certain bluest level, while for Alonso et al. (1999) the behaviour is much more regular. Taking this as the "true" behaviour, von Braun et al. (1998) RGBs would be too red for the lowest metallicities by about $0.04-0.06 \mathrm{mag}$, i.e. ages would be too high by the corresponding age difference of 2-3 Gyr, just as

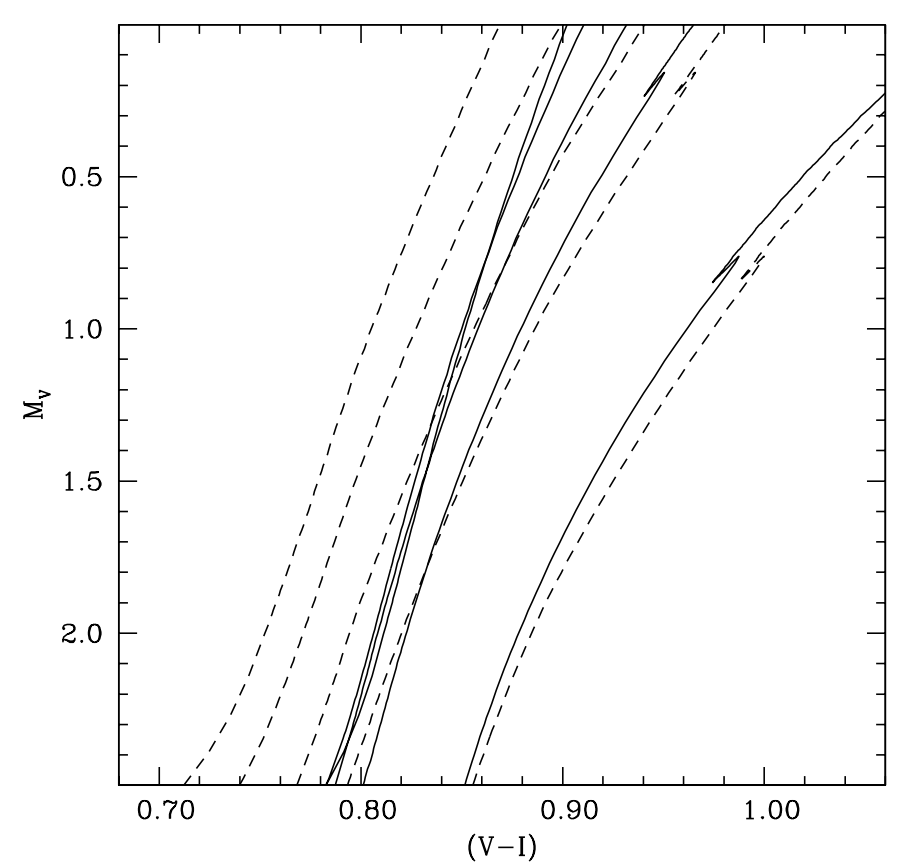

Fig. 7. Comparison of RGB $(V-I)$ colours for isochrones of 12 Gyr and $[\mathrm{Fe} / \mathrm{H}]=-2.3,-2.0,-1.6,-1.3,-0.7$ (from left to right). Solid lines correspond to the colour transformation selected in Weiss \& Salaris (1999), dashed ones to those by Alonso et al. (1999).

visible in Fig. 6. Note that at higher metallicities the two transformations agree well. Repeating therefore the horizontal absolute age determination, but using the transformation by Alonso et al. (1999), we obtain the results displayed in Fig. 8. While there is still a small age gradient across the main group of clusters, the total age difference is reduced to within $1.5 \mathrm{Gyr}$, and is consistent within the errors with the results of the AM-method - which, as already discussed in the previous section, are unaffected by the colour transformation choice - and from the $\Delta V$. This shows that our isochrones together with the colour transformations by Alonso et al. (1999) for the RGB are able to produce stable relative ages for the whole metallicity range of GCs independent of the use of the AM-method, the $\Delta V$, or $\delta C$ age indicator.

\section{Discussion}

Having fully assessed the consistency of the age distribution within our cluster sample, the results displayed in Fig. 3 can be used to investigate the formation mechanism of the Galaxy. In the following we update the discussion in SW97 and SW98, taking into account this much larger cluster sample.

There are about 150 globular clusters known in the Galaxy (Harris 1996), and probably the total population is of at most about 200 objects (Harris 1976); therefore, our sample contains between $25 \%$ and $35 \%$ of the Galactic globular cluster population, and should provide statistically more significant data about the Galaxy formation mechanism, with respect to previous works. 


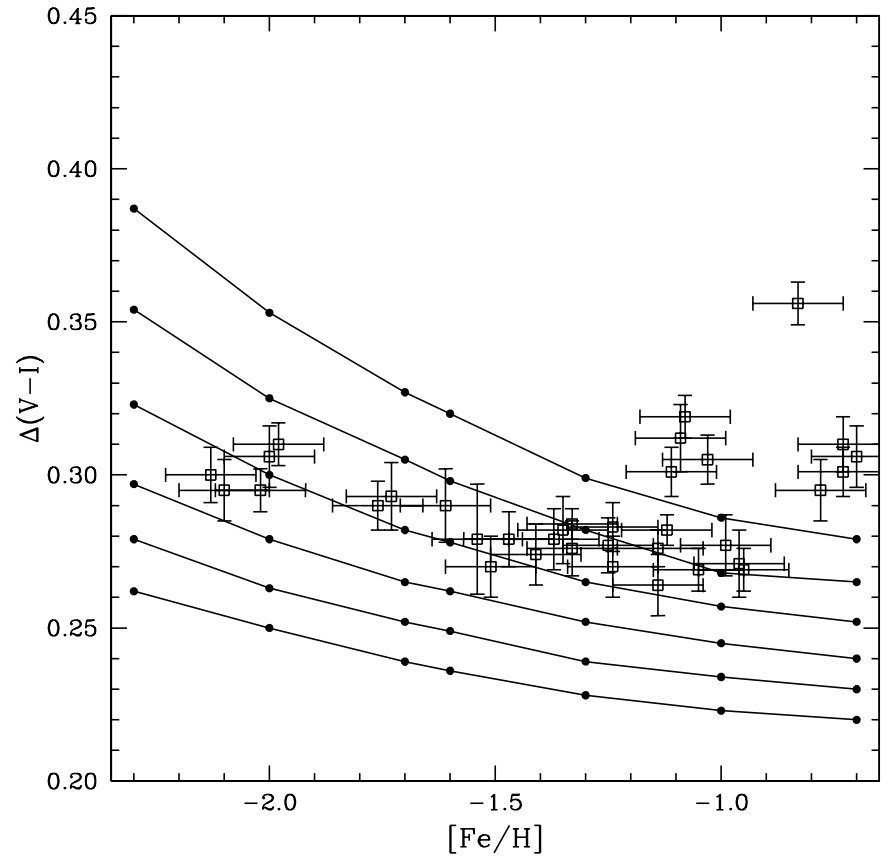

Fig. 8. As Fig. 6, but with the colour transformations by Alonso et al. (1999).

Two fundamental pieces of information need to be extracted from our age-analysis, namely the age distribution of the clusters with respect to their initial chemical composition (whose diagnostic is $[\mathrm{Fe} / \mathrm{H}]$ ), and with respect to their position within the Galaxy (whose diagnostic is $R_{\mathrm{gc}}$ ). To detect an intrinsic age spread - that is, not due to the error bar on the age determinations - among a given sample of objects which shows an age dispersion $\sigma_{\text {obs }}$, we have followed the same procedure as in SW97 (see also Chaboyer et al. 1996). More in detail, we calculate an "expected" distribution for the assumption of no intrinsic age range $\left(\sigma_{\text {range }}=0\right)$ by randomly generating, for each object, 10000 ages using a Gaussian distribution. The mean of the distribution is given by the mean age of the clusters in the given sample, and the $\sigma_{\exp }$ by the typical error in the individual age determinations (in this case we consider the total error given in Table 1, since we compare ages across multiple metallicity ranges). This is repeated for all clusters in the sample, and the F-test is then applied to the observed age distribution and this synthetic one, to test if they both show the same variance. We state that an age range exists if the probability that the two distributions have the same variance is smaller than $5 \%$. If this is true, the intrinsic age dispersion is then estimated by $\sigma_{\text {range }}=\left(\sigma_{\text {obs }}^{2}-\sigma_{\text {exp }}^{2}\right)^{0.5}$.

\subsection{Age-metallicity relationship}

When considering the complete sample of 55 objects, we obtain that all clusters with $[\mathrm{Fe} / \mathrm{H}] \leq-1.2$ result to be coeval (within the stated error bars), with an average age $\langle t\rangle=11.7 \pm 0.7 \mathrm{Gyr}$, where the dispersion of $0.7 \mathrm{Gyr}$ is entirely due to the errors in the age determination. In case of $[\mathrm{Fe} / \mathrm{H}] \geq-1.2$ a statistically significant age spread is present. The average age is $\langle t\rangle=10.1 \mathrm{Gyr}$, with an intrinsic spread $\sigma_{\text {range }}=1.9$ Gyr. In this same $[\mathrm{Fe} / \mathrm{H}]$ range we have detected, overimposed to the age spread, a marginally significant age gradient $\delta t / \delta[\mathrm{Fe} / \mathrm{H}]=-2.4 \pm$ $1.8 \mathrm{Gyr} / \mathrm{dex}$.

Our complete sample contains 3 clusters generally considered to be part of the thick disk of the Galaxy (NGC 104, NGC 6352, NGC 6838), and 2 clusters possibly belonging to the bulge population (NGC 6637, NGC 6324). All of these five clusters are metal rich and located in the inner halo. If we repeat our previous analysis excluding these 5 objects, and therefore considering only bona fide halo clusters, we obtain an average age $\langle t\rangle=$ 10.0 Gyr for the metal rich group, with $\sigma_{\text {range }}=2.5 \mathrm{Gyr}$, and a more significant $\delta t / \delta[\mathrm{Fe} / \mathrm{H}]=-4.3 \pm 2.4 \mathrm{Gyr} /$ dex.

\subsection{Age-galactocentric distance relationship}

The clusters in the so-called inner halo $\left(R_{\mathrm{gc}} \leq 8 \mathrm{kpc}\right)$ appear to be coeval, with $\langle t\rangle=11.4 \pm 0.8 \mathrm{Gyr}$, while the outer halo clusters display an intrinsic age range $\sigma_{\text {range }}=2.5$ Gyr. The average age of the outer halo sample is $\langle t\rangle=10.7 \mathrm{Gyr}$, lower than the inner halo one, simply due to the age spread towards lower ages, but there is no significant age gradient in the outer halo. This would imply that globular clusters have started forming at the same time throughout the Galaxy - this holds of course in case the actual $R_{\mathrm{gc}}$ is a good approximation of the value at the beginning of Galactic formation; see, e.g., the discussion by Searle \& Zinn (1978) on this subject - with younger and more metal rich objects being formed or possibly accreted only at large $R_{\mathrm{gc}}$ values. This scenario is very similar to the one proposed by Searle \& Zinn (1978), where the inner halo collapsed on short timescales, while the outer halo underwent a much longer formation period, accreting material over several Gyr.

When considering only bona fide halo clusters the inner halo appears still to be coeval, its average age being basically unchanged $(\langle t\rangle=11.7 \pm 0.6 \mathrm{Gyr})$.

\subsection{Influence of the sample size and metallicity scale}

The previously described results about the age distribution with respect to $[\mathrm{Fe} / \mathrm{H}]$ and $R_{\mathrm{gc}}$ are unchanged if we restrict our analysis to the more homogeneous sample of 35 clusters from R99. Moreover, the age trends with respect to $[\mathrm{Fe} / \mathrm{H}]$ and $R_{\mathrm{gc}}$ we derive are similar to the results obtained by R99 with their different technique, and statistically more significant because of the larger cluster sample. When comparing our new results with SW98 we find that the general picture of age versus $[\mathrm{Fe} / \mathrm{H}]$ is not greatly modified. In our previous works we obtained an approximate constant age for the oldest clusters at all metallicities, plus the onset of an age spread above a given $[\mathrm{Fe} / \mathrm{H}]$. However, while in SW97 and SW98 we did not find any clear correlation between age and $R_{\mathrm{gc}}$, we find now a 
difference between the age distribution of the inner and outer halo clusters. This is due to the much larger cluster sample and also to the upward revision of the age of NGC 6652, which in SW97 and SW98 resulted to be much younger than the other inner halo clusters.

The details of the scenario emerging from our age determination are however dependent on the selected $[\mathrm{Fe} / \mathrm{H}]$ scale. When we consider the ages obtained with the ZW84 scale displayed in Fig. 4, we find that only clusters with $[\mathrm{Fe} / \mathrm{H}] \leq-1.6$ are coeval, with an average age $\langle t\rangle=$ $12.2 \pm 0.6 \mathrm{Gyr}$, slightly larger than in the case of the CG97 metallicities. For larger $[\mathrm{Fe} / \mathrm{H}]$ values an intrinsic spread $\sigma_{\text {range }}=2.1 \mathrm{Gyr}$ is present, with an average age $\langle t\rangle=10.5 \mathrm{Gyr}$, and $\delta t / \delta[\mathrm{Fe} / \mathrm{H}]=-1.3 \pm 0.7 \mathrm{Gyr} / \mathrm{dex}$. Also the distribution of ages as a function of $R_{\mathrm{gc}}$ is more homogeneous, with an intrinsic age spread appearing only when $R_{\mathrm{gc}}$ is larger than $\sim 13 \mathrm{kpc}$. If we restrict the analysis to only bona fide halo clusters, we find for $[\mathrm{Fe} / \mathrm{H}]>-1.6$ a slope $\delta t / \delta[\mathrm{Fe} / \mathrm{H}]=-2.3 \pm 1.1 \mathrm{Gyr} / \mathrm{dex}$, the significance of which now exceeds the $2 \sigma$ level.

These differences come obviously from the different metallicity distribution of the clusters. In particular, in the range $-1.8 \leq[\mathrm{Fe} / \mathrm{H}] \leq-1.0$, large differences (up to $\sim 0.3$ dex) exist between the two scales.

Taking into account the effect of the uncertainty in the $[\mathrm{Fe} / \mathrm{H}]$ scale and the cluster sample size, helps in explaining discrepant conclusions reached by different authors about the existence and the onset of the age spread in the cluster population. As a test, we have considered the clusters used by VandenBerg (2000, V00) in his recent analysis; by applying our statistical analysis to his age determinations, we find the onset of an age variation among clusters starting at $[\mathrm{Fe} / \mathrm{H}] \sim-1.6$, lower than the value $[\mathrm{Fe} / \mathrm{H}]=-1.2$ found by $\mathrm{R} 99$, who used the CG97 scale, and also lower than our results when using the CG97 scale. A significant age-metallicity relationship is found for $[\mathrm{Fe} / \mathrm{H}] \geq-1.6$.

V00 derived ages for 26 objects -25 of which are also included in our sample - by employing cluster $[\mathrm{Fe} / \mathrm{H}]$ values close to the ZW84 scale, and absolute age determinations based essentially on $\Delta V$ (more precisely, the distance modulus is determined from fitting the appropriate theoretical ZAHB to the observational counterpart, and then the age is determined by the isochrone which best fits all the TO region of the observed Colour-MagnitudeDiagram).

We have rederived the ages for the 25 clusters in common assuming $\mathrm{V} 00[\mathrm{Fe} / \mathrm{H}]$ values, our isochrones, our method and our adopted photometries (which are different from the data employed by V00 for the majority of the clusters), and the results are displayed in Fig. 9, together with V00 ages shifted by -1 Gyr. When this shift of the absolute age is applied - which reflects mainly the ZAHB brightness difference between the different sets of models employed - the age distribution appears quite similar, all pairs of values being in agreement within the respective error bar. This consistency is also confirmed by the application of a K-S test to the two samples. If we restrict the

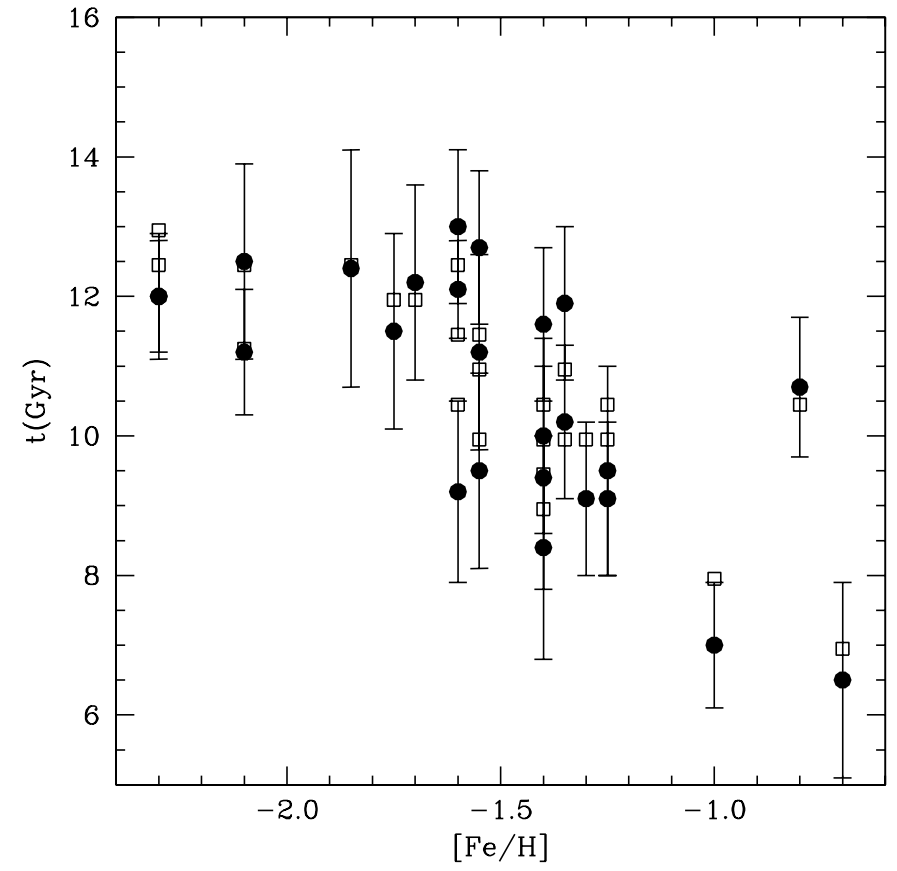

Fig. 9. Comparison between the ages derived with the AMmethod, our isochrones and our adopted photometries (filled circles), and the ages from V00 (open squares) for 25 clusters in common; an offset by -1 Gyr has been applied to the V00 data. For the sake of clarity V00 error bars (of the order of 0.8 Gyr) have been omitted.

analysis to bona fide halo clusters (thus not considering 47 Tuc in the V00 sample), for $[\mathrm{Fe} / \mathrm{H}] \geq-1.6$ we obtain an age-metallicity relationship with slope $\delta t / \delta[\mathrm{Fe} / \mathrm{H}]=$ $-4.7 \pm 0.8 \mathrm{Gyr} / \mathrm{dex}$, in good agreement with the value $\delta t / \delta[\mathrm{Fe} / \mathrm{H}]=-5.7 \pm 1.2 \mathrm{Gyr} /$ dex one obtains from V00 ages. This slope is about a factor of two higher than the one we obtained with our full sample of bona fide halo clusters coupled with the ZW84 $[\mathrm{Fe} / \mathrm{H}]$ scale.

\subsection{Second parameter and age}

With our large cluster sample it is also possible to investigate the global correlation between $[\mathrm{Fe} / \mathrm{H}], \mathrm{HB}$ type and age for the Galactic globular cluster system as a whole. We have already discussed in Sect. 2 two specific pairs of second-parameter clusters; in case of NGC 288-NGC 362 we found that age could be the main reason for the different HB types, while we do not find a significant age difference for the pair M 3-M 13.

In order to detect possible correlations between age and $\mathrm{HB}$ type (defined as $\mathrm{HB}_{\text {type }}=(B-V) /(B+V+R)$, where $B, V, R$ are the number of HB stars located, respectively, at the blue side of the instability strip, in the instability strip and at its red side) superimposed on the relationship between $\mathrm{HB}$ type and $[\mathrm{Fe} / \mathrm{H}]$, we have performed the following test. We have first considered the samples of coeval clusters with $R_{\mathrm{gc}} \leq 8$ for the CG97 $[\mathrm{Fe} / \mathrm{H}]$ scale, and $R_{\mathrm{gc}} \leq 13$ for the ZW84 one; since in both cases they span the entire metallicity range covered 


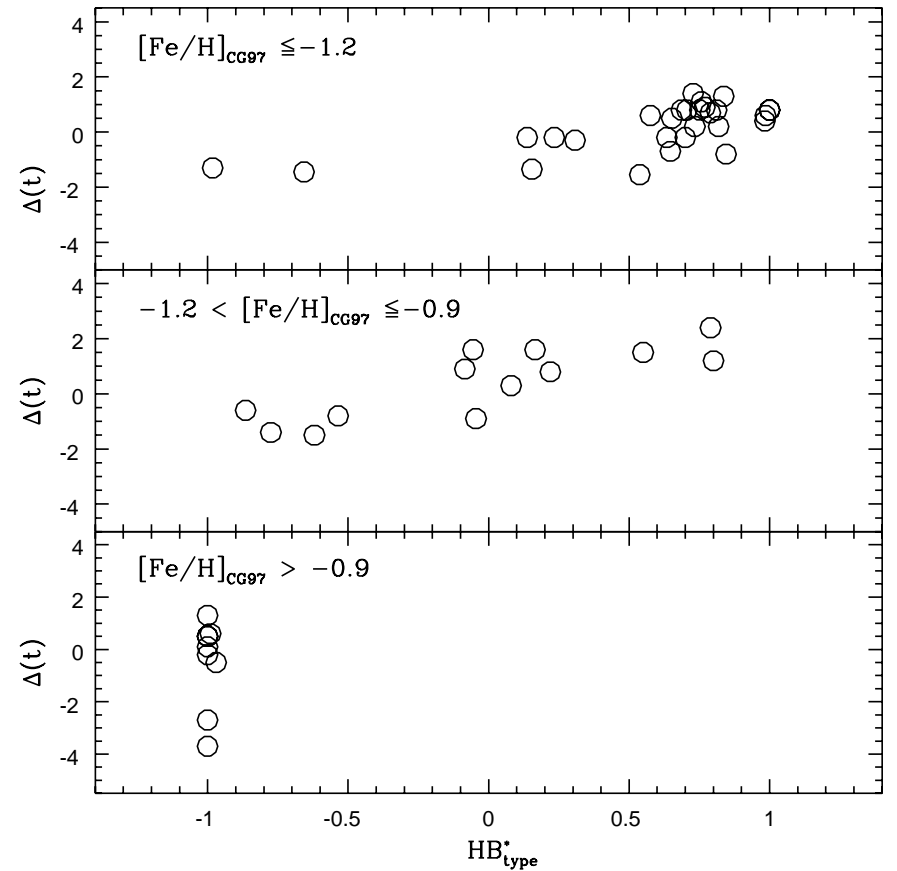

Fig. 10. Relationship between age differences $\Delta(t)$ and normalized $\mathrm{HB}$ type for clusters in the $[\mathrm{Fe} / \mathrm{H}]$ ranges (on the CG97 scale) indicated in the three panels. See text for details.

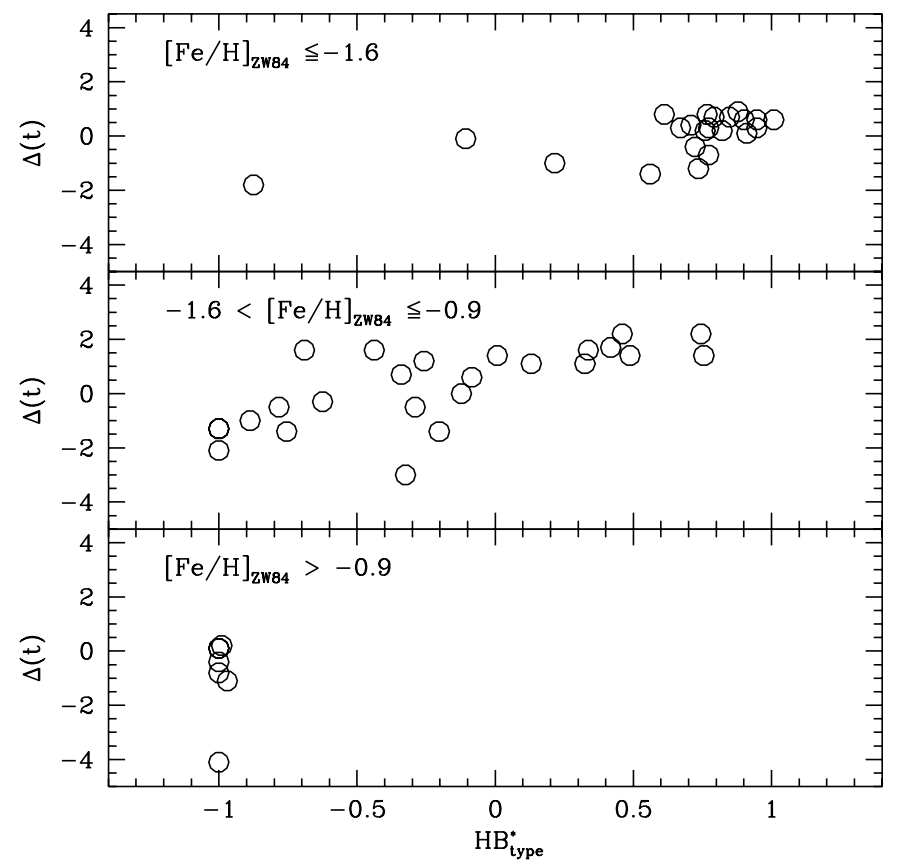

Fig. 11. As in Fig. 10 but considering the ZW84 [Fe/H] scale. See text for details

by the whole sample, we use them to determine the variation of $\mathrm{HB}_{\text {type }}$ with respect to $[\mathrm{Fe} / \mathrm{H}]$, at constant age. The entire sample has then been divided into different metallicity bins, specified in Figs. 10 and 11. For the most metal poor bin the average age of the sample has been subtracted from the individual cluster ages; in case of the two most metal rich bins, the average age of their combined sample has been subtracted from the individual values. These normalized individual ages are denoted as $\Delta(t)$. To remove the influence of the first parameter $([\mathrm{Fe} / \mathrm{H}])$, in addition, a reference $[\mathrm{Fe} / \mathrm{H}]$ has been selected for each range, and the individual values of $\mathrm{HB}_{\text {type }}$ (taken from Harris 1996 and displayed in Table 1) have been corrected using the derivative $\Delta \mathrm{HB}_{\text {type }} / \Delta[\mathrm{Fe} / \mathrm{H}]$ (determined from the previously mentioned coeval samples) and considering the difference between the actual cluster $[\mathrm{Fe} / \mathrm{H}]$ value and the reference one. By plotting $\Delta(t)$ as function of $\mathrm{HB}_{\text {type }}^{*}$, that is, the observed HB type normalized to the reference $[\mathrm{Fe} / \mathrm{H}]$ value, one should in principle be able to isolate an hypothetical correlation between age differences and $\mathrm{HB}$ colour differences, independent of the effect of $[\mathrm{Fe} / \mathrm{H}]$ variations among clusters. The reference $[\mathrm{Fe} / \mathrm{H}]$ values are, respectively, $[\mathrm{Fe} / \mathrm{H}]=-1.75$ and -1.1 for the CG97 scale, and $[\mathrm{Fe} / \mathrm{H}]=-2.0$ and -1.3 for the ZW84 one. We did not correct the HB type for the most metal rich cluster groups in Figs. 10 and 11, since the value of $\mathrm{HB}_{\text {type }}$ saturates and does not provide useful information about a possible correlation with age.

The upper panels of both Figs. 10 and 11 display $[\mathrm{Fe} / \mathrm{H}]$ ranges where our F-test analysis did not detect a significant age spread compared to the errors in the age estimates. In these ranges there is some spread in $\mathrm{HB}$ type but no significant correlation with age is found. In the middle panels the F-test analysis detected significant age spreads and also a statistically significant correlation with HB type is found. The bottom panels display clusters showing an age spread but their in this $[\mathrm{Fe} / \mathrm{H}]$ range the $\mathrm{HB}_{\text {type }}$ parameter saturates and no relevant information can be deduced.

\section{Summary}

We have determined absolute and relative ages for a large sample of globular clusters, using new and homogeneous photometric data, mostly in $V$ and $I$. We show that different age determination methods result in very similar ages, if colour transformations are carefully chosen for those methods using colour differences. Our preferred AMmethod appears however insensitive to the colour transformations choice. In a few cases, new photometric data have led to a substantial change in age compared to our previous works; this source of uncertainty should not be ignored.

From the analysis of our large sample of 55 Galactic globular clusters, we have found that:

i) Irrespective of the adopted $[\mathrm{Fe} / \mathrm{H}]$ scale, the metal poorer clusters are coeval within $\sim 1 \mathrm{Gyr}$, while an age spread appears at higher metallicites. The onset of the age spread is at $[\mathrm{Fe} / \mathrm{H}] \sim-1.2$ when using $[\mathrm{Fe} / \mathrm{H}]$ values on the CG97 scale, and $[\mathrm{Fe} / \mathrm{H}] \sim-1.6$ if one adopts the ZW84 scale.

ii) An age-metallicity relationship exists among the non-coeval clusters. In case of the CG97 scale we find $\delta t / \delta[\mathrm{Fe} / \mathrm{H}]=-2.4 \pm 1.8 \mathrm{Gyr} / \mathrm{dex}$ when considering the complete sample; if we restrict the analysis only to 
bona fide halo clusters we obtain a more significant slope $\delta t / \delta[\mathrm{Fe} / \mathrm{H}]=-4.3 \pm 2.4 \mathrm{Gyr} /$ dex. In case of the ZW84 scale we obtain $\delta t / \delta[\mathrm{Fe} / \mathrm{H}]=-1.3 \pm 0.7 \mathrm{Gyr} /$ dex for the complete sample, and $\delta t / \delta[\mathrm{Fe} / \mathrm{H}]=-2.3 \pm 1.1 \mathrm{Gyr} / \mathrm{dex}$ for the pure halo sample.

iii) Irrespective of the adopted $[\mathrm{Fe} / \mathrm{H}]$ scale, clusters closer to the galactic centre are coeval, while an age spread appears at larger galactocentric distances. In case of the CG97 $[\mathrm{Fe} / \mathrm{H}]$ scale the age spread starts at $R_{\text {gc }}$ larger than $\sim 8 \mathrm{kpc}$, while in case of the ZW84 scale it starts at $R_{\mathrm{gc}}$ above $13 \mathrm{kpc}$. In both cases there is no significant age- $R_{\mathrm{gc}}$ relationship among clusters showing an age spread.

iv) Our results yield indications that age differences are one main factor able to explain the global behaviour of the second parameter effect.

Acknowledgements. We are grateful to A. Rosenberg and I. Saviane for helpful discussions and information about the cluster sample and relative ages, and to S. Percival for a preliminary reading of the manuscript. We thank and anonymous referee for his/her comments which helped to improve the presentation of the results.

\section{References}

Alonso, A., Arribas, S., \& Martínez-Roger, C. 1999, A\&AS, 140,261

Bellazzini, M., Fusi Pecci, F., Ferraro, F. R., et al. 2001, AJ, 122,2569

Bessell, M. S., Castelli, F., \& Plez, B. 1998, A\&A, 333, 231; A\&A (Erratum), 337, 321

Buonanno, R., Corsi, C. E., Pulone, L., Fusi Pecci, F., \& Bellazzini, M. 1998, A\&A, 333, 505

Carretta, E., Gratton, R., Clementini, G., \& Fusi Pecci, F. 2000, ApJ, 215

Carretta, E., \& Gratton, R. G. 1997, A\&AS, 121, 95

Cassisi, S., Castellani, V., Degl'Innocenti, S., \& Weiss, A. 1998, A\&AS, 129, 267

Catelan, M., Bellazzini, M., Landsman, W. B., et al. 2001, AJ, 122,3171

Chaboyer, B. 2001, in ASP Conf. Ser., 245, Astrophysical Ages and Timescales, ed. T. von Hippel, C. Simpson, \& N. Manset, Astron. Soc. of the Pacific (San Francisco: Astron. Soc. of the Pacific), 162-172

Chaboyer, B., Demarque, P., \& Sarajedini, A. 1996, ApJ, 459, 558

Chaboyer, B., Sarajedini, A., \& Armandroff, T. E. 2000, AJ, 120,3102

Cote, P., Richer, H. B., \& Fahlman, G. G. 1991, AJ, 102, 1358

Harris, W. E. 1976, AJ, 81, 1095
Harris, W. E. 1996, AJ, 112, 1487

Harris, W. E., Bell, R. A., VandenBerg, D. A., et al. 1997, AJ, 114,1030

Hatzidimitriou, D., Papadakis, I., Croke, B. F. W., et al. 1999, AJ, 117, 3059

Heasley, J. N., Janes, K. A., Zinn, R., et al. 2000, AJ, 120, 879

Paltrinieri, B., Ferraro, F. R., Carretta, E., \& Fusi Pecci, F. 1998, MNRAS, 293, 434

Paltrinieri, B., Ferraro, F. R., Paresce, F., \& De Marchi, G. 2001, AJ, 121, 3114

Piotto, G., Zoccali, M., King, I. R., et al. 1999, AJ, 117, 264

Pulone, L., Salaris, M., Weiss, A., \& Buonanno, R. 1998, A\&A, 336, L77

Rey, S., Yoon, S., Lee, Y., Chaboyer, B., \& Sarajedini, A. 2001, AJ, 122, 3219

Richer, H. B., \& Fahlman, G. G. 1986, ApJ, 304, 273

Richer, H. B., Fahlman, G. G., \& Vandenberg, D. A. 1988, ApJ, 329, 187

Richer, H. B., Harris, W. E., Fahlman, G. G., et al. 1996, ApJ, 463, 602

Rosenberg, A., Saviane, I., Piotto, G., \& Aparicio, A. 1999, AJ, 118, 2306

Rosenberg, A., Aparicio, A., Saviane, I., \& Piotto, G. 2000a, A\&AS, 145, 451

Rosenberg, A., Piotto, G., Saviane, I., \& Aparicio, A. 2000b, A\&AS, 144, 5

Rutledge, G. A., Hesser, J. E., \& Stetson, P. B. 1997, PASP, 109, 907

Salaris, M., Degl'Innocenti, S., \& Weiss, A. 1997, ApJ, 479, 665

Salaris, M., \& Weiss, A. 1997, A\&A, 327, 107

Salaris, M., \& Weiss, A. 1998, A\&A, 335, 943

Sarajedini, A., \& Da Costa, G. S. 1991, AJ, 102, 628

Sarajedini, A., \& Forrester, W. L. 1995, AJ, 109, 1112

Searle, L., \& Zinn, R. 1978, ApJ, 225, 357

Smith, G. H., McClure, R. D., Stetson, P. B., Hesser, J. E., \& Bell, R. A. 1986, AJ, 91, 842

Stetson, P. B., Bolte, M., Harris, W. E., et al. 1999, AJ, 117, 247

Straniero, O., Chieffi, A., \& Limongi, M. 1997, ApJ, 490, 425

Testa, V., Corsi, C. E., Andreuzzi, G., et al. 2001, AJ, 121, 916

VandenBerg, D. A. 2000, ApJS, 129, 315

VandenBerg, D. A., Bolte, M., \& Stetson, P. B. 1990, AJ, 100, 445

VandenBerg, D. A., Stetson, F. J., Rogers, F. J., Iglesias, C. A., \& Alexander, D. R. 2000, ApJ, 532, 430

von Braun, K., Chiboucas, K., Minske, J. K., Salgado, J., \& Worthey, G. 1998, PASP, 110, 810

Weiss, A., \& Salaris, M. 1999, A\&A, 346, 897

Yim, H., Byun, Y., Sohn, Y., \& Chun, M. 2000, AJ, 120, 872

Zinn, R., \& West, M. J. 1984, ApJS, 55, 45 